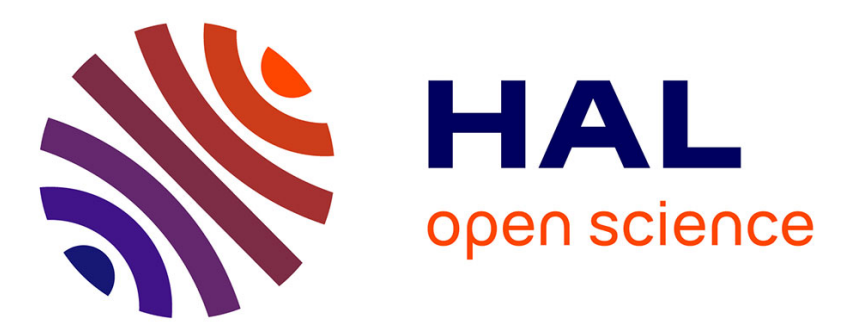

\title{
Le RS3PE syndrome ou polyarthrite aiguë œdémateuse bénigne du sujet âgé
}

Daniel Wendling, Frank Verhoeven, Clément Prati

\section{To cite this version:}

Daniel Wendling, Frank Verhoeven, Clément Prati. Le RS3PE syndrome ou polyarthrite aiguë œedémateuse bénigne du sujet âgé. Revue du Rhumatisme monographies, 2019, 86, pp.195 - 198. 10.1016/j.monrhu.2018.10.005 . hal-03486571

\section{HAL Id: hal-03486571 \\ https://hal.science/hal-03486571}

Submitted on 20 Dec 2021

HAL is a multi-disciplinary open access archive for the deposit and dissemination of scientific research documents, whether they are published or not. The documents may come from teaching and research institutions in France or abroad, or from public or private research centers.
L'archive ouverte pluridisciplinaire HAL, est destinée au dépôt et à la diffusion de documents scientifiques de niveau recherche, publiés ou non, émanant des établissements d'enseignement et de recherche français ou étrangers, des laboratoires publics ou privés.

\section{다(1) $\$$}

Distributed under a Creative Commons Attribution - NonCommercial| 4.0 International 


\section{Le RS3PE syndrome ou polyarthrite aiguë œdémateuse bénigne du sujet âgé}

RS3PE syndrome : remitting seronegative symmetrical synovitis with pitting edema

Daniel Wendling $(1,2)^{*}$, Frank Verhoeven $(1,3)$, Clément Prati $(1,3)$,

(1) Service de Rhumatologie, CHU Minjoz, 25030 Besançon

(2) EA 4266 (EPILAB) Université de Franche-Comté, Besançon

(3) EA 4267 (PEPITE) Université de Franche-Comté, Besançon

*Correspondance : Daniel Wendling, Service de rhumatologie, CHU Minjoz, Boulevard Fleming, 25030 Besançon.

dwendling@chu-besancon.fr 
Résumé :

Individualisé en 1985 par Mc Carty, le RS3PE syndrome (ou polyarthrite aiguë œéémateuse bénigne du sujet âgé) réalise un tableau de polysynovite avec œdème distal. Touchant avec prédilection le sujet de plus de 65 ans, son évolution est habituellement favorable dans un délai de 6 à 12 mois sous corticothérapie à faible dose. Les circonstances de diagnostic et le cadre nosologique de ce syndrome se sont élargis, faisant du RS3PE un mode de début potentiel de maladies systémiques inflammatoires du sujet âgé.

Mots-clés : RS3PE syndrome, polyarthrite, sujet âgé

Abstract :

In 1985 Mc Carthy described the RS3PE syndrome (remitting symmetrical seronegative synovitis with pitting edema). It occurs predominantly in males over 65 years with favorable outcome in a 6 to 12 months delay under moderate steroids dosage. Diagnosis circumstances and nosologic frame enlarged over time, positioning RS3PE syndrome as a potential mode of onset of systemic inflammatory diseases in the elderly.

Key words : RS3PE syndrome, polyarthritis, elderly people 


\section{Introduction.}

C'est en 1985 que Mc Carty, à propos de dix cas, propose d'individualiser sous l'acronyme RS3PE (remitting, seronegative, symmetrical synovitis with pitting edema) un tableau de synovite symétrique des membres supérieurs associé à un œè̀me du dos des mains [1]. Ce tableau, proche de la polyarthrite rhumatoïde (PR), s'en distingue par quelques particularités cliniques et évolutives.

\section{Clinique}

Ce syndrome touche avec une prédominance masculine (2/3) les sujets de plus de 65 ans. Le début est le plus souvent brutal, l'œdème est le premier symptôme, précédent parfois jusqu'à plusieurs semaines la polyarthrite ou polysynovite bilatérale et symétrique des articulations métacarpo-phalangiennes et interphalangiennes proximales et des poignets. Des ténosynovites des fléchisseurs des doigts (pouvant être à l'origine d'un syndrome du canal carpien) ou des extenseurs sont très fréquemment associées. L'atteinte des pieds et des chevilles est moins constante ; l'atteinte des épaules, des coudes ou des genoux est plus rare.

Les œdèmes représentent une caractéristique : ils sont distaux, bilatéraux, blancs, mous et prennent le godet. Ils peuvent parfois être volumineux réalisant un aspect « en gant de boxe » (Figure 1). Ils sont constants aux membres supérieurs et associés aux membres inférieurs dans 2/3 des cas environ (Figure 2).

Des signes généraux peuvent être associés : asthénie, fébricule, amaigrissement. Présents à la phase initiale, ils restent modérés et non spécifiques. [2]

\section{Paraclinique.}

Sur le plan biologique, un syndrome inflammatoire souvent marqué est régulièrement observé, parfois associé à une anémie et une hypo albuminémie. Il n'y a pas de marqueur biologique spécifique. La recherche de facteur rhumatoïde est constamment négative; les anticorps anti nucléaires sont absents. L'étude des antigènes d'histocompatibilité a mis en évidence une association avec HLA B7 dans près de $60 \%$ des cas (incidence dans la population générale caucasienne : $24 \%$ ) dans la série initiale de Mc Carthy [1], mais non confirmée dans d'autres séries [3,4]. Il n'a pas été mis en évidence de lien avec HLA DR [5]. Le liquide synovial a pu être analysé dans certains cas, révélant un liquide peu inflammatoire ; la biopsie synoviale met en évidence une inflammation non spécifique. 
Certains marqueurs biologiques ont été plus particulièrement évalués dans ce syndrome. C'est le cas notamment du vascular endothelial growth factor (VEGF). Des taux sériques élevés de VEGF ont été mis en évidence chez les patients atteints de RS3PE syndrome avant traitement comparativement à des sujets sains, avec normalisation sous traitement corticoïde [6]. Cependant, lors d'une étude rétrospective de rhumatismes inflammatoires du sujet âgé (n $=80)$, si les taux de VEGF sont globalement plus élevés que chez les sujets sains $(\mathrm{n}=37)$, il n'a pas été observé de différence entre RS3PE et autres rhumatismes inflammatoires du sujet âgé, ni d'association entre le taux de VEGF et la présence d'œdème distal [7].

D'autres travaux ont soulevé un intérêt potentiel pour l'évaluation des métalloprotéases matricielles (MMP-3) dans le sérum de patients [8], en particulier dans les formes paranéoplasiques [9]. Ces résultats nécessitent confirmation.

La radiographie des sites articulaires touchés n'objective pas de modifications spécifiques ; des érosions sont signalées dans 5,5\% des cas dans l'analyse systématique des 331 cas de la littérature en 2016 [2]. Les techniques actuelles d'imagerie (échographie, IRM) visualisent l'œdème sous cutané, les synovites et ténosynovites, avec une hypervascularisation de ces structures [10]. Au TEP scanner, parfois réalisé à la recherche d'une néoplasie profonde ou d'une atteinte vasculaire (artérite à cellules géantes) ou encore de bursites d'une PPR, les aspects de RS3PE sont représentés par une captation diffuse, habituellement symétrique des tissus mous péri articulaires [11].

\section{Traitement}

Les anti-inflammatoires non stéroïdiens peuvent avoir une efficacité sur les manifestations synovitiques [1], mais leur tolérance chez le sujet âgé en limite l'utilisation. La corticothérapie générale à doses moyennes $(16.12 \pm 9,5 \mathrm{mg} /$ jour de prednisone dans l'analyse de la littérature) [2] a démontré une efficacité souvent spectaculaire sur l'ensemble des signes, faisant régresser l'œdème et la CRP en quelques jours, et les synovites plus progressivement. Les traitements de fond des rhumatismes inflammatoires sont rarement nécessaires du fait de l'efficacité habituelle et rapide de la corticothérapie ; l'hydroxychloroquine a été utilisée dans certains cas [1]. L'évolution est favorable, le plus souvent en 6 mois, permettant l'arrêt de la corticothérapie. La possibilité de persistance d'un enraidissement indolore des doigts à distance de la phase aiguë doit être signalée. 


\section{Situation nosologique}

Il s'agit très clairement d'une association syndromique, dans laquelle les œdèmes distaux occupent une place clinique importante.

Ainsi, devant un tableau de rhumatisme inflammatoire, les œè̀mes feront discuter, après avoir éliminé une cause rénale, cardiaque ou hépatique (où les œdèmes peuvent être bilatéraux et diffus), la possibilité d'une thrombose veineuse (Syndrome des anti phospholipides, Maladie de Behcet), ou une conséquence d'un kyste poplité ; dans ces cas, l'œdème est unilatéral et localisé à une extrémité le plus souvent. Cependant une atteinte RS3PE unilatérale peut être observée, en particulier en cas d'hémiparésie (côté indemne d'œdème) [12].

L'association œdèmes et douleurs ostéo articulaires peut correspondre à d'autres situations. C'est le cas des algodystrophies, qui sont le plus souvent unilatérales ; l'œdème est associé à des troubles vaso moteurs, des modifications de coloration cutanée, une hyperthermie locale à la phase initiale, associées à une hyperalgésie. L'absence de syndrome inflammatoire biologique est un élément important de la discussion diagnostique. Les examens d'imagerie (scintigraphie, IRM aux stades précoces, radiologie au stade avancé), mettent en évidence les modifications caractéristiques de l'algodystrophie.

L'ostéoarthropathie hypertrophiante pneumique, ou syndrome de Pierre-Marie, s'accompagne parfois de douleurs et d'œdèmes distaux. Les éléments cliniques de dysacromélie (hippocratisme digital) et les modifications radiologiques (appositions périostées) permettent de redresser le diagnostic.

Certaines formes de rhumatisme inflammatoire à début tardif peuvent prêter à confusion (tableau I) et sont sujets à discussion dans les modalités évolutives du syndrome.

Dans le cadre des spondylarthropathies, les auteurs clermontois [13] ont décrit le tableau de «Late Onset Peripheral Spondylarthropathy » ou LOPS qui est un tableau de spondylarthropathie à début tardif (plus de 60 ans) avec atteinte périphérique. Dans ce tableau à prédominance masculine, des œdèmes sont notés dans plus de $3 / 4$ des cas, mous, prenant le godet. Cependant l'atteinte prédomine aux membres inférieurs (les mains sont plus rarement touchées), et l'atteinte articulaire est une oligoarthrite asymétrique. Une arthrite sacro iliaque peut compléter le tableau dans un cas sur deux, et l'atteinte rachidienne est modérée. Les signes généraux sont habituellement marqués. Biologiquement, un syndrome inflammatoire franc est habituel, et il est retrouvé une liaison avec HLA-B27. Cette forme tardive de spondylarthropathie se caractérise par la faible efficacité des AINS et des corticoïdes; les 
modifications radiologiques de ces localisations sont tardives et d'interprétation difficile chez le sujet âgé. L'évolution est chronique sur plusieurs années avec la possibilité de rechutes.

Le rhumatisme psoriasique peut se manifester par des œdèmes des mains, le plus souvent asymétrique, en association avec les ténosynovites et les dactylites (doigt saucisse). L'œdème, dans ce cas est dur et ne prend pas le godet. L'association au RS3PE a été rapportée [14].

La polyarthrite rhumatoïde peut, de façon peu fréquente, s'accompagner d'œdèmes des extrémités, souvent asymétriques. Ce sont des œdèmes lymphatiques survenant plutôt sur des PR anciennes et évoluées, leur évolution n'est pas forcément parallèle aux poussées articulaires de la maladie. Dans le cas de PR à début tardif, le diagnostic peut être particulièrement difficile. Le syndrome inflammatoire biologique est souvent marqué, la présence de facteurs rhumatoïdes ou d'anticorps anti CCP orientent le diagnostic, de même que la mise en évidence d'images d'érosions osseuse en radiologie et surtout en échographie et en IRM, plus précoces. Si le diagnostic entre RS3PE et PR à début tardif peut parfois être difficile, des différences immuogénétiques et de répartition des sous populations lymphocytaires [15] argumentent en faveur de deux maladies différentes [16].

La pseudopolyarthrite rhizomélique (PPR) représente le diagnostic différentiel majeur devant un tableau d'atteinte inflammatoire de l'appareil locomoteur de début brutal chez une personne de plus de 60 ans, avec signes généraux et syndrome inflammatoire biologique. La PPR est plus fréquente que le RS3PE, mais des atteintes périphériques sont décrites dans environ $25 \%$ des cas de PPR, et des œdèmes distaux prenant le godet ont été signalés dans $10 \%$ des cas de PPR dans certaines séries. La réponse favorable à la corticothérapie générale dans les deux cas ne permet pas la distinction. Le suivi prospectif de 23 cas de RS3PE et 177 cas de PPR consécutifs a mis en évidence une similitude des aspects démographiques, cliniques, biologiques, évolutifs sous traitement et IRM entre les deux conditions, suggérant une communauté des deux entités, avec un meilleur pronostic en cas d'œdème périphérique [17]. De même il a été observé des modifications identiques de sous populations lymphocytaires entre PPR et RS3PE [15], et dans une autre étude comparative de 28 RS3PE et 123 PPR, les caractéristiques des 2 groupes étaient similaires, mais les patients RS3PE sont plus volontiers de sexe masculin, à tendance dépressive et tabagique [18].

D’autres diagnostics peuvent être discutés chez la personne âgée dans cette situation :

Les arthropathies microcristallines associent inflammation aiguë clinique et biologique, la possibilité de signes généraux, et des phénomènes d'œdèmes locaux [19]. Le plus souvent localisés à une région articulaire (poignet), ils peuvent parfois avoir une présentation polyarticulaire. C'est surtout la chondrocalcinose articulaire qui est en cause, le diagnostic 
étant orienté par la constatation radiologique des calcifications typiques (du ligament triangulaire du carpe en particulier) ; plus rarement c'est une goutte qui est en cause.

L'amylose [20], les formes œdémateuses de sclérodermie, la sarcoïdose [21], le syndrome de Shulman, les polymyosites peuvent également associer des œdèmes aux manifestations ostéoarticulaires, et ont leurs caractéristiques cliniques et paracliniques permettant de redresser le diagnostic.

\section{Associations et aspects évolutifs.}

Depuis les descriptions initiales, des cas d'association de RS3PE à d'autres pathologies, et des évolutions vers certaines maladies inflammatoires ont fait reconsidérer la place nosologique de ce syndrome.

Ainsi, il a été rapporté l'association de RS3PE avec différents cas de vascularite et de maladies systémiques (LED) [22] et avec des pathologies évoquées dans le diagnostic différentiel (spondylarthropathies, amylose, sarcoïdose...), faisant envisager le RS3PE comme mode de début non spécifique de certaines maladies du sujet âgé.

L'association à des cancers (en particulier adénocarcinomes prostatiques, digestifs) ou à des hémopathies (lymphomes, syndromes myélodysplasiques) a été rapportée à plusieurs reprises (16\% des cas dans la revue de la littérature en 2016 [2]), soulevant la question du caractère paranéoplasique du RS3PE dans cette situation [9,23,24]. Dans ce cas, les signes généraux sont plus marqués, et la corticosensibilité moins nette. Dans quelques observations, les œdèmes ont disparu après exérèse de la tumeur.

Associations particulières.

Des cas isolés de survenue de RS3PE syndrome dans des situations particulières font discuter la possibilité de certains agents inducteurs.

C'est le cas de certains agents infectieux tels le parvo virus B19 [25]. Des agents pharmacologiques ont également été incriminés : BCG intravésical [26], rifampicine [27], anti-diabétiques [28], et récemment les inhibiteurs de check point, en particulier le Nivolumab (anti PD-1), avec régression des symptômes cliniques et biologiques sous corticothérapie et maintien du traitement anti cancéreux [29,30].

Si l'évolution du RS3PE est classiquement favorable sous traitement en 6 à 12 mois, le suivi de certains patients a mis en évidence l'apparition de signes cliniques et paracliniques d'autres maladies. C'est ainsi que, à côté de la possibilité de rechutes, certains cas ont évolué secondairement vers une spondylarthropathie, une PR, une PPR [31], un syndrome de Gougerot-Sjögren [32]. Dans la série bordelaise, sur 24 cas, la moitié évolue dans le suivi vers 
une autre pathologie inflammatoire définie [33]. Le suivi de 41 patients japonais durant un an illustre une moins bonne réponse à la corticothérapie en cas de sexe masculin et de CRP initiale élevée [34].

\section{Physiopathologie.}

Elle a été peu étudiée et reste du domaine des hypothèses. La diminution des cellules $\mathrm{T}$ suppressives/cytotoxique et l'augmentation des cellules Th1 circulantes [15] sont susceptibles de favoriser le développement de l'inflammation. D'autre part, la constatation de taux de VEGF plus élevés que chez les sujets sains est également à prendre en compte. Le VEGF induit l'inflammation synoviale et la perméabilité vasculaire [6] ; ceci peut rendre compte des phénomènes d'œdème distal si particulier à ce syndrome.

\section{Conclusion}

Ainsi, l'ensemble de ces données fait discuter la place de ce syndrome qui peut exister de façon primitive, d'évolution favorable et limitée dans le temps sous traitement corticoïde ; le rapprochement avec la PPR, dont il pourrait représenter une forme clinque, est tentant.

Ce syndrome pourrait également, dans certains cas, représenter un mode de début, non spécifique, de différentes pathologies du sujet âgé, rhumatologiques ou générales (y compris néoplasiques), à envisager en particulier en cas de non-réponse à la corticothérapie à faible dose ou en cas de récidive. Dans ces situations, des explorations complémentaires pouvant aller jusqu'à la tomographie par émission de positons.

L'apparition des éléments cliniques d'un RS3PE syndrome justifie un bilan initial clinique et paraclinique (bilan inflammatoire, hémogramme, recherche d'auto-anticorps, électrophorèse des protéines, radiographies articulaires, imagerie thoraco abdominale) de même qu'un suivi prolongé de ces patients.

Les auteurs ne déclarent pas de conflit d'intérêt concernant cet article. 


\section{Références :}

1. McCarty DJ, O'Duffy JD, Pearson L, Hunter JB. Remitting seronegative symmetrical synovitis with pitting edema. RS3PE syndrome. JAMA. 1985;254:27637.

2. Karmacharya P, Donato AA, Aryal MR,et al. RS3PE revisited: a systematic review and meta-analysis of 331 cases. Clin Exp Rheumatol. 2016 ;34(3):404-15.

3. Cantini F, Salvarani C, Olivieri I,et al. Remitting seronegative symmetrical synovitis with pitting oedema (RS3PE) syndrome: a prospective follow up and magnetic resonance imaging study. Ann Rheum Dis. 1999;58:230-6.

4. Pease C, Bhakta B. RS3PE syndrome. The relation with HLA B7. Ann Rheum Dis. $2000 ; 59: 239-40$.

5. Toussirot E, Berthier S, Wendling D, Tiberghien P. Lack of association between HLA DRB1* alleles and RS3PE syndrome. Ann Rheum Dis 1998;57:442

6. Arima K, Origuchi T, Tamai M, et al. RS3PE syndrome presenting as vascular endothelial growth factor associated disorder. Ann Rheum Dis. 2005;64:1653-5.

7. Smets P, Devauchelle-Pensec V, Rouzaire PO, Pereira B, Andre M, Soubrier M. Vascular endothelial growth factor levels and rheumatic diseases of the elderly. Arthritis Res Ther. 2016;18:283.

8. Kenzaka T, Goda K. Serum matrix metalloproteinase 3 in detecting remitting seronegative symmetrical synovitis with pitting edema syndrome: A case report. World J Clin Cases. 2018;6:84-87.

9. Origuchi T, Arima K, Kawashiri SY, et al. High serum matrix metalloproteinase 3 is characteristic of patients with paraneoplastic remitting seronegative symmetrical synovitis with pitting edema syndrome. Mod Rheumatol. 2012;22:584-8.

10. Klauser A, Frauscher F, Halpern E, et al. Remitting seronegative symmetrical synovitis with pitting edema of the hands: ultrasound, color doppler ultrasound, and magnetic resonance imaging findings. Arthritis Rheum 2005;53:226-33

11. Wang J, Pu C, Wang Z, Yang J. Remitting Seronegative Symmetrical Synovitis With Pitting Edema: Appearance on FDG PET/CT. Clin Nucl Med. 2017 ;42:492-495.

12. Keenan RT, Hamalian GM, Pillinger MH. RS3PE presenting in a unilateral pattern: case report and review of the literature. Semin Arthritis Rheum. 2009 ;38:428-33. 
13. Dubost JJ, Sauvezie B. Late onset peripheral spondyloarthropathy. J Rheumatol $1989 ; 16: 1214-7$

14. Kardes S, Karagulle M, Erdogan N. Remitting seronegative symmetrical synovitis with pitting oedema (RS3PE) associated with psoriatic arthritis. Scand J Rheumatol. 2015;44(4):339-40.

15. Shimojima Y, Matsuda M, Ishii W, Gono T, Ikeda S. Analysis of peripheral blood lymphocytes using flow cytometry in polymyalgia rheumatica, RS3PE and early rheumatoid arthritis. Clin Exp Rheumatol. 2008;26:1079-82.

16. Yao Q, Su X, Altman RD. Is remitting seronegative symmetrical synovitis with pitting edema (RS3PE) a subset of rheumatoid arthritis? Semin Arthritis Rheum. 2010;40:89-94.

17. Cantini F, Salvarani C, Olivieri I, et al. Remitting seronegative symmetrical synovitis with pitting oedema (RS3PE) syndrome: a prospective follow up and magnetic resonance imaging study. Ann Rheum Dis. 1999;58:230-6

18. Kimura M, Tokuda Y, Oshiawa H, et al. Clinical characteristics of patients with remitting seronegative symmetrical synovitis with pitting edema compared to patients with pure polymyalgia rheumatica. J Rheumatol. 2012;39:148-53.

19. Palazzi C, Olivieri I, D'Amico E, Pace-Palitti V, Petricca A. Symmetrical pitting edema resembling RS3PE in gout. Clin Rheumatol. 2003;22:506-7.

20. Magy N, Michel F, Augé B, Toussirot E, Wendling D. Amyloid arthropathy revealed by RS3PE syndrome. Joint Bone Spine 2000;67:475-7

21. Matsuda M, Sakurai K, Fushimi T, et al.Sarcoidosis with high serum levels of vascular endothelial growth factor (VEGF), showing RS3PE-like symptoms in extremities. Clin Rheumatol. 2004;23:246-8.

22. Hegazi MO, Saleh F, Al Rashidi A, Yaktien MM. Synovitis with pitting edema as the presenting manifestation of systemic lupus erythematosus. Lupus. 2014 ;23:1069-72.

23. Sibilia J, Friess S, Schaeverbeke T, et al. Remitting seronegative symmetrical synovitis with pitting edema (RS3PE): a form of paraneoplastic polyarthritis? J Rheumatol. 1999;26:115-20.

24. Tunc SE, Arslan C, Ayvacioglu NB, Sahin M, Akkus S, Yorgancigil H. Paraneoplastic remitting seronegative symmetrical synovitis with pitting edema (RS3PE syndrome): a report of two cases and review of the literature. Rheumatol Int. 2004;24:234-7. 
25. Perandones CE, Colmegna I, Arana RM. Parvovirus B19: another agent associated with remitting seronegative symmetrical synovitis with pitting edema. $\mathbf{J}$

Rheumatol. 2005;32:389-90.

26. Mouly S, Berenbaum F, Kaplan G. Remitting seronegative symmetrical synovitis with pitting edema following intravesical bacillus Calmette-Guérin instillation.

J Rheumatol. 2001;28:1699-701.

27. Smyth D, Rehman R, Remund K, Egan J. Remitting seronegative symmetrical synovitis with pitting oedema associated with rifampicin. Ir J Med Sci. 2011 ;180:585-6.

28. Yamauchi K, Sato Y, Yamashita K, et al.

RS3PE in association with dipeptidyl peptidase-4 inhibitor: report of two cases. Diabetes Care. 2012;35:e7

29. Gauci ML, Baroudjian B, Laly P, et al. Remitting seronegative symmetrical synovitis with pitting edema (RS3PE) syndrome induced by nivolumab. Semin Arthritis Rheum. 2017;47:281-287.

30. Ngo L, Miller E, Valen P, Gertner E. Nivolumab induced remitting seronegative symmetrical synovitis with pitting edema in a patient with melanoma: a case report. J Med Case Rep. 2018;12:48.

31. Berthier S, Toussirot E, Wendling D. Polyarthrite aiguë oedémateuse bénigne du sujet âgé (ou RS3PE syndrome). Aspects évolutifs à propos de 13 cas. Presse Med $1998 ; 27: 1718-22$

32. Choi YM, Sheen DH, Lee YJ, Lee EB, Song YW. Sjogren's syndrome presenting as remitting seronegative symmetric synovitis with pitting edema (RS3PE). J Korean Med Sci. 2003;18:606-8.

33. Schaeverbeke T, Fatout E, Marcé S, et al. Remitting seronegative symmetrical synovitis with pitting oedema: disease or syndrome? Ann Rheum Dis. 1995;54:681-4.

34. Origuchi T, Arima K, Umeda M, et al. Clinical outcomes in the first year of remitting seronegative symmetrical synovitis with pitting edema (RS3PE) syndrome. Mod Rheumatol. 2017;27:150-154. 
Tableau I : caractéristiques des principaux rhumatismes inflammatoires du sujet âgé

\begin{tabular}{|l|l|l|l|l|}
\hline & PPR & Polyarthrite & RS3PE & LOPS \\
\hline Localisation & proximale & $\begin{array}{l}\text { Proximale et } \\
\text { périph. }\end{array}$ & périphérique & $\begin{array}{l}\text { Périphérique + } \\
\text { axiale }\end{array}$ \\
\hline Oedèmes & + & + & +++ mains & +++ pieds \\
\hline Evolution & 0 séquelle & Destruction & Possibilité & Possibilité \\
articulaire & contractures & destruction \\
\hline HLA & DR 4 & DR 4 & B7 & B 27 \\
\hline Traitement & corticoïdes & Corticoïdes + & corticoïdes & Inefficacité \\
& & Tt de fond & & corticoïdes \\
\hline Délai guérison & 18 mois & Années & 12 mois & Années \\
\hline
\end{tabular}


Figure 1 : œdème du dos de la main, prenant le godet

Figure 2 : œdème du dos du pied 


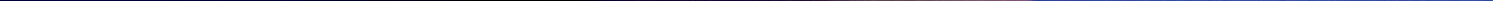

